\title{
PREPARAÇÃO E CARACTERIZAÇÃO DO COMPLEXO DE INCLUSÃO DO ÓLEO ESSENCIAL DE Croton zehntneri COM $\beta$-CICLODEXTRINA
}

\author{
Ulisses N. Aguiara, Sidney G. de Lima ${ }^{\mathrm{a}, \mathrm{b}, *}$, Márcio S. Rocha ${ }^{\mathrm{a}}$, Rivelilson Mendes de Freitas ${ }^{\mathrm{a}}$, Talita M. Oliveira ${ }^{\mathrm{a}}$, Raiane M. \\ Silva ${ }^{b}$, Lara C. B. Mourab e Lorena T. G. de Almeida ${ }^{b}$ \\ aDepartamento de Bioquímica e Farmacologia, Universidade Federal do Piauí, Campus Universitário Ministro Petrônio Portella, \\ s/n, 64049-550 Teresina - PI, Brasil \\ bDepartamento de Química, Universidade Federal do Piauí, Campus Universitário Ministro Petrônio Portella, s/n, 64049-550 \\ Teresina - PI, Brasil
}

Recebido em 21/03/2013; aceito em 11/08/2013; publicado na web em 24/09/2013

\begin{abstract}
PREPARATION AND CHARACTERIZATION OF THE INCLUSION COMPLEX ESSENTIAL OIL OF Croton zehntneri WITH $\beta$-CYCLODEXTRIN. Croton zehntneri, a plant native to northeastern Brazil, is widely used in folk medicine to treat gastrointestinal problems and has rich essential oil content. The essential oil of $C$. Zehntneri was analyzed by GC-MS, and its inclusion complex with $\beta$-cyclodextrin ( $\beta$-CD) was characterized by both vibrational spectroscopy and differential scanning calorimetry (DSC). Estragol was the major component identified in the essential oil by the study. IR spectra indicated an interaction of $\beta$-CD with essential oil from C. zehntneri, a finding corroborated by the stability constant and scanning calorimetry. Microencapsulation within $\beta$-CD has the potential to mask sensory attributes and increase aqueous solubility of oils, thereby improving their applicability as drugs.
\end{abstract}

Keywords: Croton zehntneri; essential oil; $\beta$-cyclodextrin.

\section{INTRODUÇÃO}

A espécie Croton zehntneri Pax et Hoffm, conhecida popularmente como canela de cunhã, canelinha ou canela-brava, pertence à família Euforbiaceae, é usada comumente na medicina popular principalmente como sedativo, estimulante de apetite e para aliviar distúrbios intestinais, sendo comprovados os efeitos antinociceptivos de seus óleos essenciais. ${ }^{1}$ É uma planta subarbustiva e caducifólia, sendo que o seu aroma varia entre exemplares desta planta coletados em diferentes regiões do Nordeste. Isto se deve à variação na concentração dos constituintes químicos mais abundantes nos seus óleos essenciais. ${ }^{2}$ Assim, distinguem-se para esta espécie quatro tipos químicos como: anetol - para os exemplares coletados em Fortaleza (CE) e Viçosa (CE); eugenol - para os coletados em Areia Branca (RN) e Quixadá (CE); metil-eugenol - para os coletados em Ipu (CE) e Oeiras (PI); estragol - Tianguá (CE) e Granja (CE). ${ }^{3}$

Por estarem presentes em diversas partes das plantas (produzidos pelo metabolismo), possuírem o seu potencial farmacológico comprovado em estudos científicos, por serem de fácil extração e economicamente viáveis, os óleos essenciais têm assumido papel de destaque nas pesquisas em produtos naturais. Seus constituintes variam desde hidrocarbonetos terpênicos, álcoois simples e terpênicos, aldeídos, cetonas, fenóis, ésteres, óxidos, peróxidos, furanos, ácidos orgânicos, lactonas, cumarinas, entre outros, sendo que a grande maioria é constituída de derivados fenilpropanóides ou de terpenóides, principalmente de monoterpenos e sesquiterpenos. ${ }^{4}$ Os óleos essenciais das espécies do C. zenhtneri e C. nepetaefolius apresentam na sua constituição monoterpenóides e sesquiterpenóides, como também fenilpropanóides. O C. argyrophylloides apresentam monoterpenóides e sesquiterpenóides, mas não contém fenilpropanóides. ${ }^{3}$ Resultados publicados sobre o óleo essencial de espécies do gênero Croton verificaram, na espécie C. zenhtneri, o estragol $(76,8 \%)$ como composto majoritário. ${ }^{5}$

$\mathrm{O}$ estragol, um dos principais constituintes do óleo essencial

*e-mail: sidney@ufpi.edu.br de C. zenhtneri, caracteriza-se por ser um éter fenilpropanóide, designado 4-metoxi-2-propenilbenzeno (Figura 1). ${ }^{6}$ Foi atribuído ao estragol, componente ativo do óleo essencial de Enterolobium contortisiliquum (Leguminosae), a responsabilidade pela atividade antimicrobiana desse óleo. ${ }^{7}$

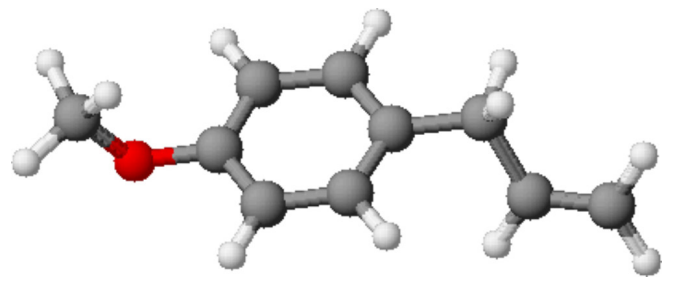

Figura 1. Estrutura do estragol (modelo bolas e varetas), principal constituinte do óleo essencial de Croton zehntneri, optimizada em programa ACD/3D: ChemSketch

Dessa substância já foram descritas atividades biológicas, tais como: antimicrobiana, anti-inflamatória e antioxidante, o que o credencia como constituinte para um possível fármaco. Foram demonstradas também atividade antinociceptiva ${ }^{8}$ e depressora sobre o sistema nervoso central. ${ }^{9}$ Considerando suas propriedades físico-químicas, é uma substância que se apresenta líquida à temperatura ambiente, de aspecto oleoso, incolor ou levemente amarelado. Possui baixa estabilidade quando exposto ao ar, umidade, luz, calor e metal. ${ }^{4}$ Entretanto, sua instabilidade térmica, sua menor solubilidade em água e sua elevada volatilidade dificultam aplicabilidade tecnológica. Essas características podem ser melhoradas através da formação do complexo de inclusão do óleo essencial de $C$. zenhtneri com $\beta$-Ciclodextrina $(\mathrm{OEC} / \beta-\mathrm{CD})$.

As ciclodextrinas (CDs) são oligossacarídeos cíclicos, obtidos a partir de degradação enzimática do amido pela enzima ciclodextrina glicosiltransferase, ${ }^{10,11}$ produzida por diferentes microrganismos, como o Bacillus macerans, B. megaterium, B. circulans, B. stearothermophilus, B. alcalofílico, B. subtilis, Micrococcus varians, 
M. luteus e Klebsiella pneumoniae. ${ }^{12}$ As mais comuns são as $\alpha, \beta$ e $\gamma$-ciclodextrinas, compostas por 6, 7 ou 8 unidades de glicopiranose. ${ }^{10}$ As ciclodextrinas apresentam conformação espacial tronco cônica, com grupos hidroxilas primários e secundários voltados para a face externa, conferindo certa hidrofilia para a superfície externa, enquanto a cavidade da molécula é hidrofóbica. ${ }^{13}$ Destas a $\beta-C D$ é a mais utilizada, pois sua cavidade pode hospedar moléculas de massa molecular entre 100 e $400 \mathrm{~g} / \mathrm{mol}$, faixa de massa molecular da maioria das moléculas de interesse. ${ }^{12,14}$ Além disso, o preço razoável desta ciclodextrina também propicia seu uso. ${ }^{15}$ As ciclodextrinas são solúveis em água e possuem a capacidade de formar complexos de inclusão reversíveis com moléculas apolares, incrementando de forma exponencial sua solubilidade em meio aquoso. ${ }^{12}$

Baseado nesta propriedade, diversos óleos essenciais e compostos voláteis passaram a ser complexados com ciclodextrinas, ${ }^{13,14,16-22}$ aumentando sua estabilidade, solubilidade aquosa, fotoestabilidade e estabilidade térmica, além de protegê-los da oxidação, mascarar ou reduzir efeitos fisiológicos indesejados, além de reduzir sua volatilidade. $^{15}$

Apesar do grande número de trabalhos desenvolvidos na linha de pesquisa de compostos de inclusão com CDs, a caracterização dos complexos constitui, ainda hoje, uma etapa delicada no processo de síntese. ${ }^{10,13}$

Neste trabalho foi avaliada a preparação e análise do complexo de inclusão do óleo essencial de $C$. zenhtneri, coletado em Simões (PI), com $\beta$-ciclodextrina, usando técnicas espectroscópicas. A escolha do desenvolvimento desse trabalho, inicialmente com C. zenhtneri, é devido a sua ampla presença no município e região circunvizinha e à extensa e potencial aplicação terapêutica do componente estragol, substância majoritária dessa planta, de fácil extração e baixo custo de produção.

\section{PARTE EXPERIMENTAL}

\section{Materiais e métodos}

\section{Material vegetal}

Folhas de Croton zehntneri Pax et Hoffm (Euforbiaceae), foram coletadas em fevereiro de 2011 no município de Simões-PI, Brasil, para extração dos constituintes voláteis. Uma amostra representativa foi identificada e depositada no Herbário Graziela Veloso da Universidade Federal do Piauí, sob o número 29.131.

\section{Extração dos óleos essenciais}

O óleo essencial das folhas frescas de C. zenhtneri (OEC) foi extraído continuamente durante 3 horas em um aparelho tipo Clevenger ${ }^{\circledR}$, seco com sulfato de sódio anidro, pesado e armazenado em frasco tampado, protegido com papel alumínio e armazenado em refrigerador sob temperatura média de $4{ }^{\circ} \mathrm{C}$ até o momento das análises.

\section{Análise por cromatografia gasosa}

A análise da amostra de óleo foi realizada por Cromatografia Gasosa acoplada à Espectrometria de Massas (CG-EM) utilizando equipamento Hewlett-Packard, modelo 5890 SERIES II e coluna capilar apolar DB-5 de sílica fundida (30 m x 0,25 mm i.d., película de $0,25 \mu \mathrm{m})$. O gás de arraste utilizado foi o hélio, sob o de fluxo 1 $\mathrm{mL} / \mathrm{min}$ e modo de divisão $1 / 10$. A temperatura do injetor e do detector foi mantida em $250{ }^{\circ} \mathrm{C}$ e $270{ }^{\circ} \mathrm{C}$, respectivamente. A temperatura da coluna foi programada de $50{ }^{\circ} \mathrm{C}$ a $180{ }^{\circ} \mathrm{C}$ a uma taxa de $4{ }^{\circ} \mathrm{C} / \mathrm{min}$ e em seguida $180^{\circ} \mathrm{C}$ a $260^{\circ} \mathrm{C}$ a uma taxa de $10^{\circ} \mathrm{C} / \mathrm{min}$, permanecendo 10 minutos a $260^{\circ} \mathrm{C}$. Os espectros de massas foram gravados na faixa de 30 a $450 \mathrm{~m} / \mathrm{z}$ e o volume injetado foi de $1 \mu \mathrm{L}$.
Os componentes individuais foram identificados por correspondência de seus espectros de massa, obtidos com fonte de ionização por impacto de elétrons a $70 \mathrm{eV}$. Uma série de C8-C20 n-alcanos foi usada como referência para o cálculo do índice de retenção, bem como por comparação visual da fragmentação padrão com aqueles relatados na literatura. A composição percentual foi obtida a partir da medida da integração das áreas sob os picos. ${ }^{23-26}$

\section{Preparo do complexo de inclusão}

O complexo de inclusão (CI) entre o óleo essencial de $C$. zenhtneri $(\mathrm{OEC})$ com $\beta$-ciclodextrina ( $\beta$-CD) foi preparado, em triplicata, de acordo com o método proposto por Bhandari e colaboradores, ${ }^{27} \mathrm{com}$ algumas modificações. Uma alíquota $25 \mathrm{mg}$ do OEC foi solubilizado em $500 \mu \mathrm{L}$ de etanol PA e adicionado a uma solução de $200 \mathrm{mg}$ de $\beta$-CD solubilizada em $10 \mathrm{~mL}$ de uma mistura etanol:água (1:2) aquecida a $55^{\circ} \mathrm{C}$. Após a adição do OEC, a mistura foi agitada a 150 rpm por 4 horas, a uma temperatura de $25^{\circ} \mathrm{C}$. Após este período, a suspensão foi retirada da agitação e resfriada a $4{ }^{\circ} \mathrm{C}$ por 12 horas. $\mathrm{O}$ precipitado foi recolhido, seco por liofilização e pesado.

\section{Preparo da mistura física}

A mistura física (MF) entre o OEC e a $\beta$-CD na razão de 1:1 foi homogeneizada com o auxílio de gral com pistilo e, posteriormente, foi armazenada em frasco hermeticamente fechado.

\section{Diagrama de solubilidade do óleo essencial de C. zenhtneri}

$\mathrm{O}$ estudo de solubilidade de fases foi realizado conforme o método descrito por Higuchi e Connors, ${ }^{28}$ adicionando-se excesso de $\mathrm{OEC}$ a tubos de ensaio contendo soluções aquosas da $\beta-\mathrm{CD}$, em concentrações crescentes $(0-10 \mathrm{mM})$. As amostras foram mantidas sob agitação, por 24 horas. Alíquotas foram retiradas, filtradas em papel filtro e adequadamente diluídas em etanol PA, mantendo-se a relação de 1 parte de água para 9 partes de etanol. O conteúdo foi determinado em espectrofotômetro (Varian AA 50) em 268 nm. Os experimentos foram realizados em triplicata.

A constante de estabilidade (Kc) foi calculada e estimou-se uma possível estequiometria de formação do complexo, a partir da solubilidade intrínseca do OEC e a inclinação da reta obtida no diagrama de solubilidade de fases, a partir da Equação $1 .^{10}$

$$
K_{1: 1}=\frac{\text { Inclinação }}{S_{0} \times(1-\text { Inclinação })}
$$

onde, $\mathrm{S}_{0}$ corresponde à solubilidade intrínseca do óleo essencial.

\section{Caracterização do complexo de inclusão}

\section{Espectroscopia de absorção no infravermelho}

Os espectros de IV do OEC, $\beta-C D, M F$ e CI foram obtidos na região do IV médio (4000 a $600 \mathrm{~cm}^{-1}$ ) em um espectrômetro Vertex 70 (Bruker), utilizando acessório para Reflectância Total Atenuada (ATR). Cerca de $20 \mu \mathrm{L}$ de OEC, $5 \mathrm{mg}$ de $\beta$-CD, MF e CI foram colocadas diretamente sobre a superfície do cristal $(\mathrm{Ge})$ do acessório de ATR, aplicando uma ligeira pressão para promover um contato entre as amostras e os sólidos na superfície do cristal. Os espectros foram obtidos a partir de 64 varreduras e uma resolução de $4 \mathrm{~cm}^{-1}$. Os espectros de IV foram suavizados e a linha de base foi corrigida automaticamente utilizando o software do espectrofotômetro.

\section{Calorimetria exploratória diferencial (DSC)}

A análise térmica por DSC (2920 TA Instruments) foi realizada em atmosfera de nitrogênio com fluxo de $50 \mathrm{~mL} / \mathrm{min}$, empregando uma massa de aproximadamente $5,5 \mathrm{mg}$, acondicionada em 
porta-amostra de alumina hermeticamente fechado. Analisou-se o OEC, a $\beta$-CD, a MF e o CI entre 30 a $300{ }^{\circ} \mathrm{C}$, sob um razão de aquecimento de $10{ }^{\circ} \mathrm{C} \mathrm{min}^{-1}$.

\section{RESULTADOS E DISCUSSÃO}

\section{Composição química do óleo essencial de C. zenhtneri}

O rendimento do óleo, obtido a partir de $325 \mathrm{~g}$ de folhas de $C$. zehntneri, canelinha, foi de cerca de $2,0 \%$. A análise por CG-EM permitiu a identificação de $100 \%$ dos constituintes integrados. A Figura 2 mostra o perfil cromatográfico da amostra de óleo analisada por CG-EM. Foi possível identificar quatro constituintes principais, sendo o estragol $(99,15 \%)$ o principal componente. Eucaliptol, biciclogermacreno e o espatulenol aparecem em baixíssima abundância relativa.

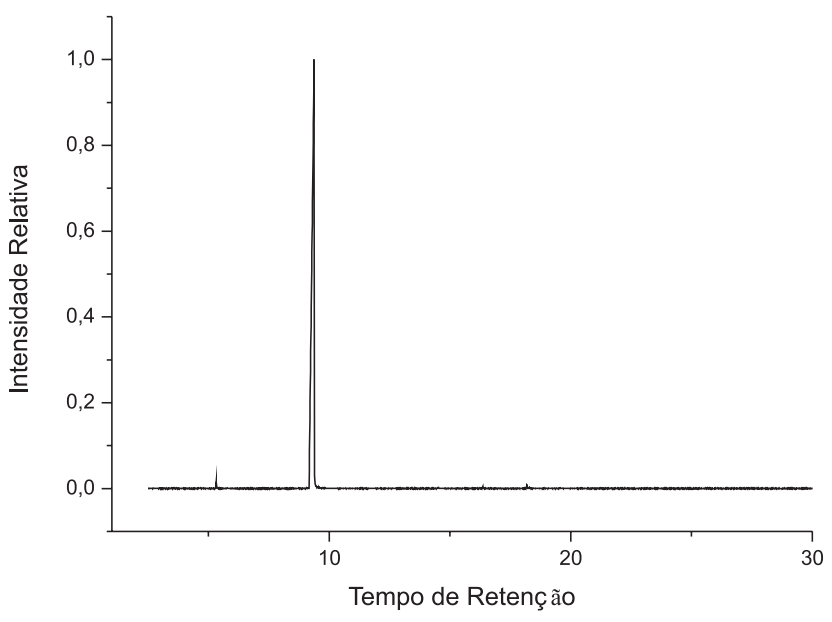

Figura 2. Perfil cromatográfico (TIC) do óleo essencial de Croton zehntneri

\section{Diagrama de solubilidade do óleo essencial de $C$. zenhtneri}

Por meio da leitura espectrofotometrica observou-se que o OEC tem um pico máximo de absorbância em $268 \mathrm{~nm}$ em etanol:água (9:1) (Figura 3), comprimento de onda a partir do qual foi construída a curva de calibração do OEC (Figura 4), obtendo-se a seguinte equação da reta: $\mathrm{Y}=0,01929 \mathrm{X}-0,3447$. Verificou-se que a $\beta-C D$ não possuía absorbância significativa na região do ultravioleta, não interferindo na leitura espectrofotométrica do OEC.

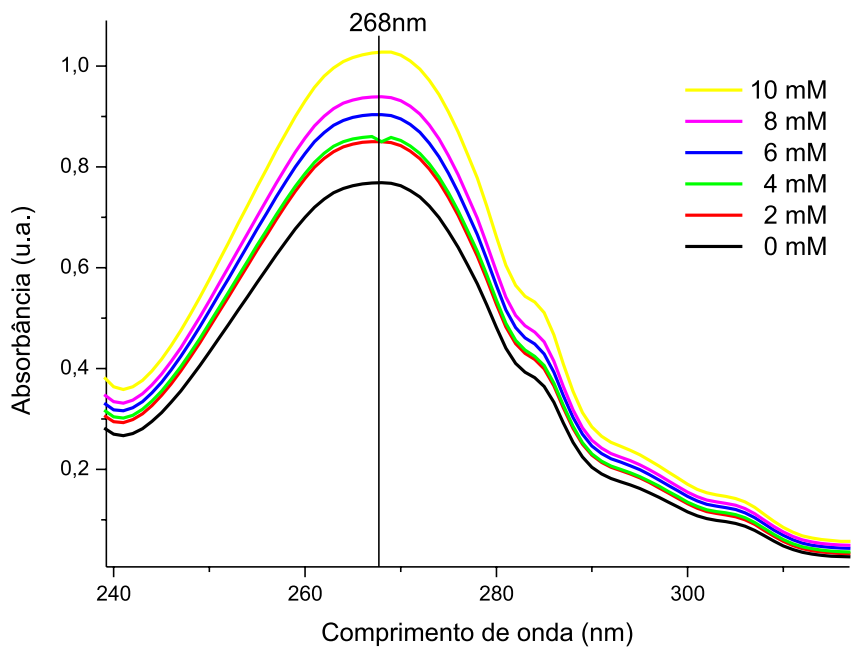

Figura 3. Leitura espectrofotometrica do óleo essencial de Croton zehntneri

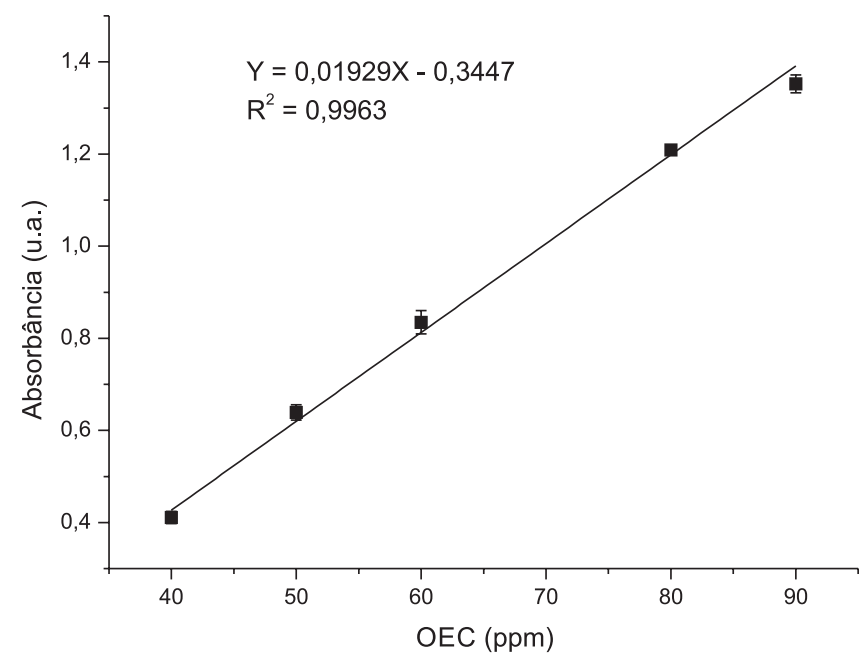

Figura 4. Curva de Calibração do óleo essencial de Croton zehntneri

A estequiometria do complexo OEC: $\beta-C D$ foi estimada pela técnica de solubilidade de fases, a partir da relação linear obtida entre a quantidade de OEC solubilizado e a concentração de $\beta$-CD das soluções (Figura 5), ${ }^{28}$ sugerindo a formação de complexo de primeira ordem relativamente a $\mathrm{CD}$ e de ordem 1 ou maior que 1 para o $\mathrm{OEC}$, ou seja, o aumento da solubilidade do OEC é proporcional ao incremento das concentrações da $\mathrm{CD}$, característico de uma estequiometria 1:1 ou superior, por exemplo, $2: 1 .^{12}$

De acordo com Veiga e colaboradores, ${ }^{12}$ a relação estequiométrica mais usual para complexos com CD é 1:1 e que a maioria das moléculas hóspedes complexadas com $\beta$-CD possuem peso molecular entre 100 e 400 Dáltons. No caso do OEC, seu constituinte majoritário é o estragol, com peso molecular de 148,2 $\mathrm{g} \mathrm{mol}^{-1}$, se enquadrando dentro da faixa usual de peso molecular para a formação do complexo com $\beta$-CD.

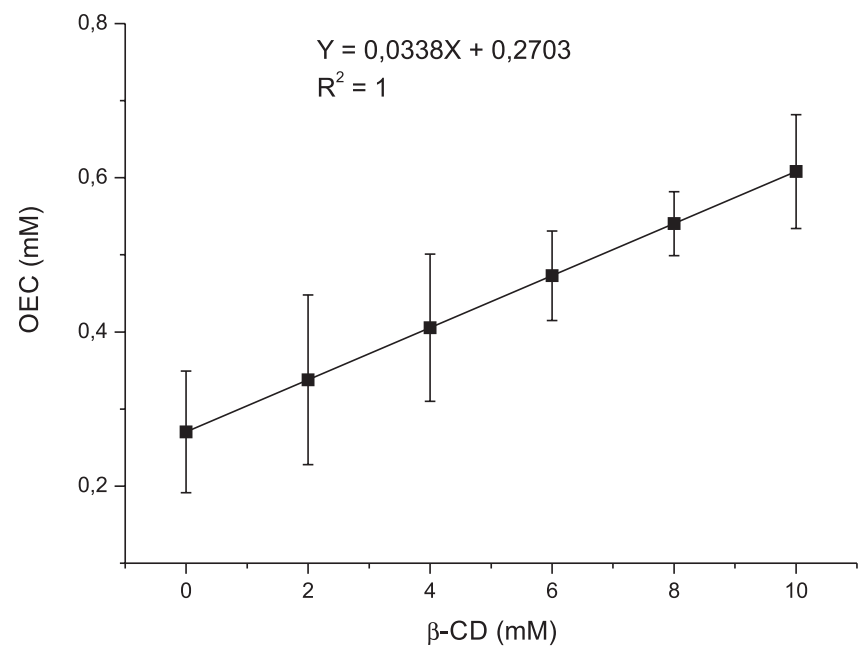

Figura 5. Diagrama de solubilidade do óleo essencial de Croton zehntneri em presença de $\beta$-ciclodextrina $(\beta-C D)$

A constante de estabilidade $(\mathrm{Kc})$ também é um parâmetro de grande interesse para a caracterização da interação molecular entre os diversos componentes do complexo e sua viabilidade prática, visto que complexos com elevada constante de estabilidade podem originar complexos muito estáveis. ${ }^{12}$ A constante de estabilidade $(\mathrm{Kc})$ do sistema OEC/ $\beta-C D$ obtido foi de 129,6 L mol-1 , indicando uma boa interação entre o OEC e a $\beta$-CD. Esse resultado está de acordo com outros complexos de inclusão obtidos, onde a $\beta$-CD hospeda, no 
interior de sua cavidade, pequenas moléculas hidrofóbicas. ${ }^{29}$ Tian e colaboradores ${ }^{18}$ preparando complexo de inclusão do óleo essencial de Salvia sclarea com $\beta$-CD, determinaram a constante de estabilidade $164,5 \mathrm{M}^{-1}$, por outro lado, Wang e colaboradores ${ }^{14}$ determinaram a constante de estabilidade do complexo do óleo de alho com $\beta$-CD como sendo $1141 \mathrm{M}^{-1}$, indicando que a força de interação entre as diferentes moléculas hóspedes e a $\beta$-CD é específica, gerando a formação de complexos com maior ou menor estabilidade. ${ }^{12}$

No entanto, deve-se buscar não apenas o melhor incremento de solubilidade, mas também a menor proporção de $\beta$-CD para solubilização do fármaco. ${ }^{30}$

\section{Preparo do complexo OEC/ $\beta$-CD}

Por sua grande eficiência e facilidade de execução em escala laboratorial, a técnica de co-precipitação no preparo do complexo de inclusão, ${ }^{18}$ combinada com sua ampla utilização para obtenção de complexo de inclusão da $\beta$-CD com óleos essenciais, ${ }^{12,14,17,18,29,30}$ foi escolhida para o preparo do complexo de inclusão OEC/ $\beta$-CD.

A formação do complexo de inclusão é facilitada pela remoção das moléculas de água do interior da cavidade parcialmente hidrofóbica da CD e sua substituição por moléculas apolares de forma espontânea, sendo, portanto, um processo energeticamente viável. ${ }^{10-12,15,18,31-34}$

A solubilidade intrínseca da molécula hóspede também é determinante neste processo ${ }^{32}$ e, por fim, como o fenômeno de complexação é um processo concentração dependente, a razão molar entre a molécula
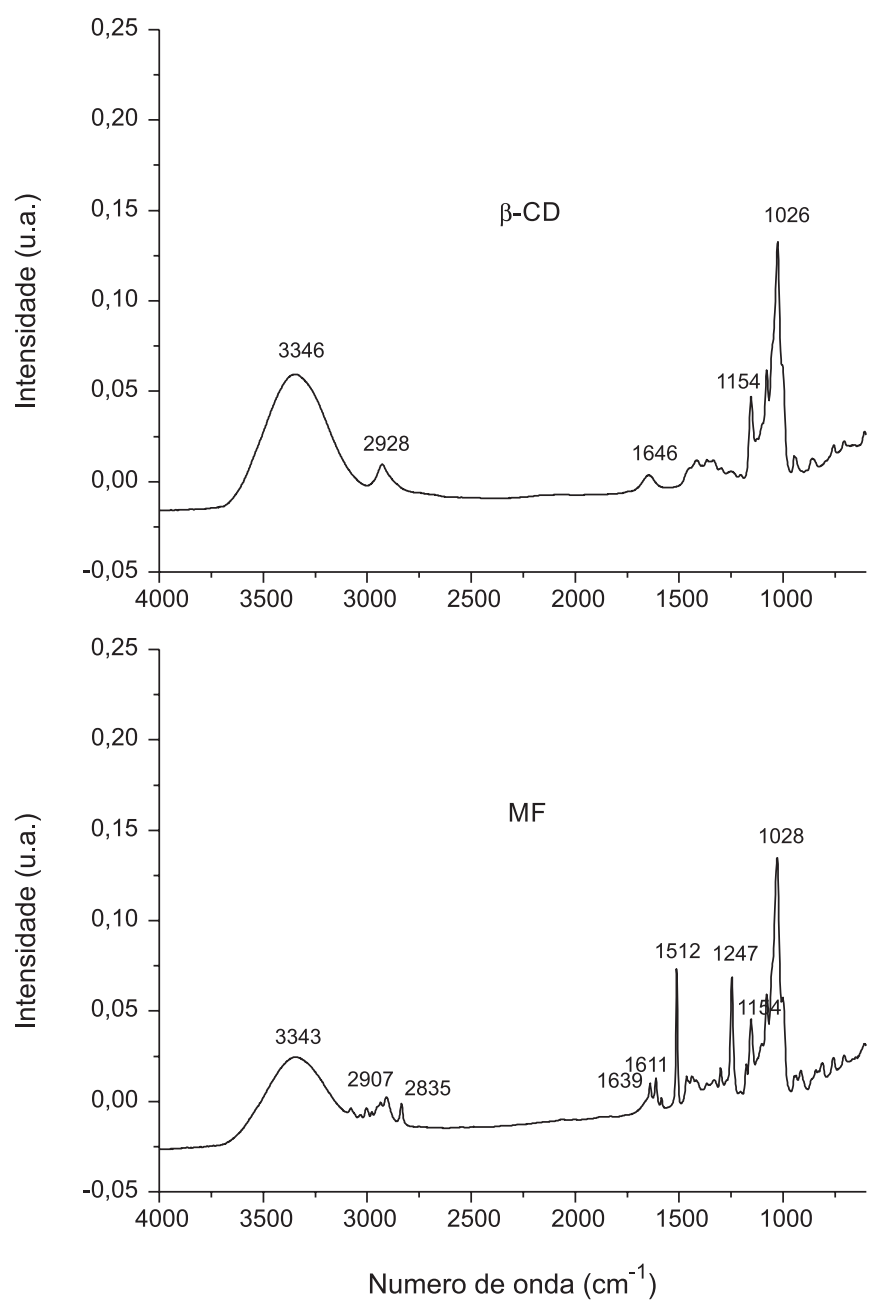

hóspede e a ciclodextrina também influencia no processo de formação dos complexos de inclusão. ${ }^{34}$

\section{Espectroscopia de infravermelho}

A espectroscopia de absorção no IV é restrita a espécies moleculares que apresentam alteração do momento de dipolo elétrico intrínseco como consequência de seu movimento vibracional ou rotacional, provocado pela absorção da energia radiante, ${ }^{35}$ sendo essa técnica analítica bastante utilizada para a avaliação da ocorrência de interações entre diferentes moléculas no estado sólido, mediante comparação das bandas da molécula hóspede e do carreador, com a mistura física e o complexo. ${ }^{36,37}$ Os espectros fornecem informações sobre a estrutura molecular, dinâmica e ambiente, ${ }^{38,39}$ sendo estas informações de grande importância na confirmação da identidade de uma substância analisada. Os espectros de infravermelho (IV) da $\beta$-CD, OEC, MF e CI são apresentados na Figura 6.

$\mathrm{O}$ espectro de infravermelho da $\beta$-CD mostrou bandas de absorção em $3346 \mathrm{~cm}^{-1}$ (estiramento $\mathrm{O}-\mathrm{H}$ ), $2928 \mathrm{~cm}^{-1}$ (estiramento $\mathrm{C}-\mathrm{H}$ ), $1154 \mathrm{~cm}^{-1}$ (estiramento $\mathrm{C}-\mathrm{O}$ ) e $1026 \mathrm{~cm}^{-1}$ (estiramento $\mathrm{C}-\mathrm{O}-\mathrm{C})$. O espectro de IV do OEC mostrou bandas de absorção entre 3077 e $2835 \mathrm{~cm}^{-1}$ (estiramento C - H), 1639 e $1611 \mathrm{~cm}^{-1}$ (estiramento $\mathrm{C}=\mathrm{C}$ ), $1510 \mathrm{~cm}^{-1}$ (estiramento do anel aromático), 1246 $\mathrm{cm}^{-1}$ (estiramento de $\mathrm{C}-\mathrm{O}$ arílico), característicos da presença do estragol. O espectro de infravermelho da MF mostra picos característicos tanto da $\beta$-CD quanto do OEC, sugerindo pouca ou nenhuma
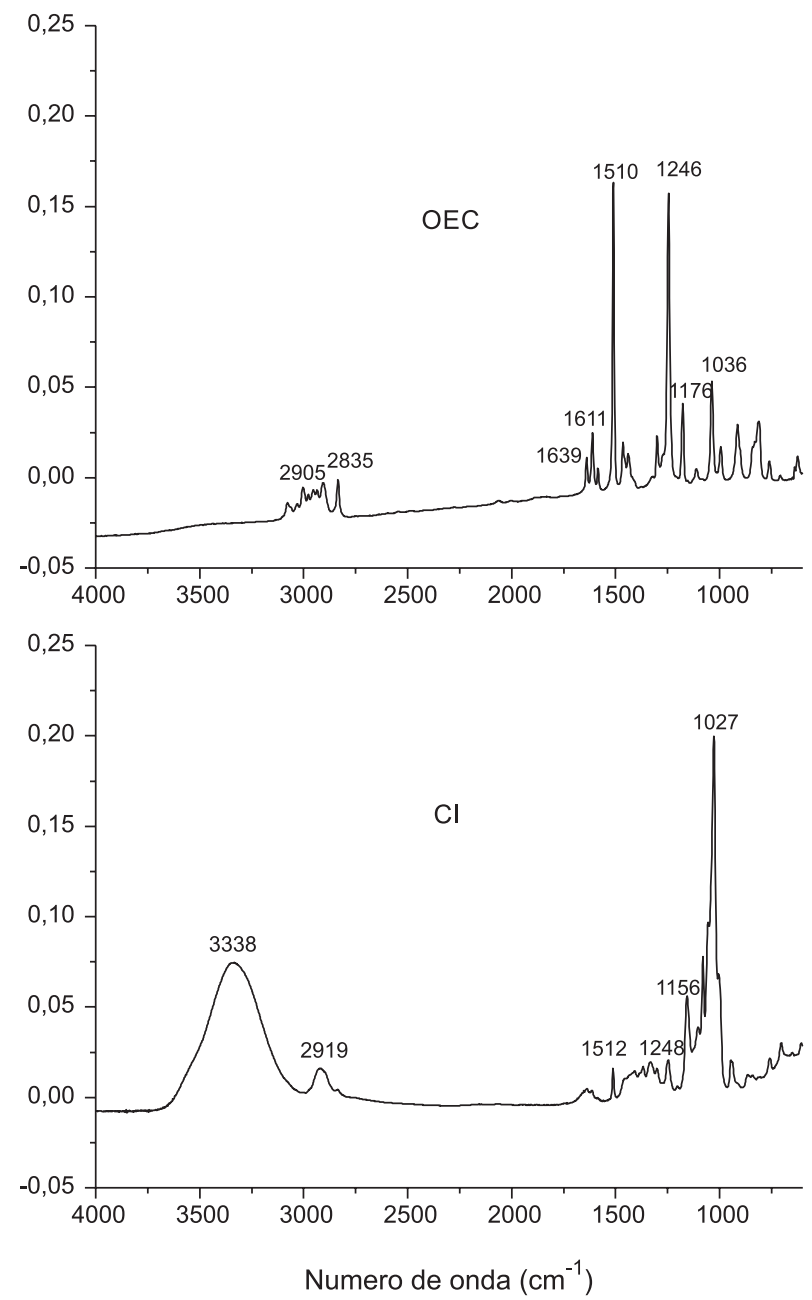

Figura 6. Espectros de Infravermelho das Amostras de $\beta$-ciclodextrina ( $\beta$-CD), Mistura Física (MF), Complexo de Inclusão (CI) e óleo essencial de Croton zehntneri $(O E C)$ 
interação entre as moléculas. No espectro do complexo de inclusão (CI) observou-se uma forte semelhança com o espectro da $\beta$-CD. Em especial, as bandas características do OEC em 2835, 1639, 1611 e $1510 \mathrm{~cm}^{-1}$ tiveram uma acentuada redução de intensidade comparada aos espectros isolados do OEC e da MF, sugerindo que estas ligações possivelmente estão implicadas na formação destes complexos, visto que a inserção de uma molécula-hóspede no interior da cavidade da $\beta$-CD provoca uma restrição conformacional, reduzindo a livre movimentação das moléculas encapsuladas, reduzindo a intensidade do sinal. Esse mesmo efeito foi percebido por Fini, ${ }^{39}$ Oliveira, ${ }^{33}$ e Rocha ${ }^{31}$ quando prepararam complexos de inclusão utilizando a $\beta$-CD. Além destes efeitos, observou-se ainda o deslocamento das bandas de estiramento $\mathrm{OH}$ da $\beta-\mathrm{CD}$ em $3346 \mathrm{~cm}^{-1}$ para $3338 \mathrm{~cm}^{-1}$ no $\mathrm{CI}$ e da banda de estiramento $\mathrm{C}-\mathrm{H}$ da $\beta$-CD em $2928 \mathrm{~cm}^{-1}$ para $2919 \mathrm{~cm}^{-1}$ no CI, reforçando a teoria da formação do complexo de inclusão.

\section{Calorimetria exploratória diferencial (DSC)}

Calorimetria exploratória diferencial (DSC) é uma das técnicas mais utilizadas para confirmar a formação do complexo de inclusão no estado sólido, devido a sua sensibilidade em detectar possíveis alterações nos eventos térmicos característicos da molécula hóspede quando esta se encontra no interior da cavidade da ciclodextrina, bem como a estabilidade do sistema e sua cristalinidade. ${ }^{10,12,14,40} \mathrm{O}$ comportamento térmico de todos os materiais foi investigado usando DSC em atmosfera inerte (Figura 7). O termograma da $\beta-\mathrm{CD}$ e da MF mostra um acentuado pico endotérmico em $120^{\circ} \mathrm{C}$. Este evento térmico, de acordo com Wang ${ }^{14}$ e Karathanos ${ }^{41}$ possivelmente é provocado pela perda de água presente no interior de sua cavidade hidrofóbica. Este pico está muito reduzido no termograma do CI, indicando um possível deslocamento das moléculas de água por moléculas de estragol, indicando que ocorreu a formação do complexo. Ainda na Figura 7 pode ser observado no termograma de DSC, característico do OEC, composto por um pico endotérmico em $108^{\circ} \mathrm{C}, 118^{\circ} \mathrm{C}$ e $190{ }^{\circ} \mathrm{C}$. A curva DSC da MF parece ser a simples superposição da curva DSC da $\beta$-CD. Entretanto, um padrão diferente foi observado na curva DSC do CI. O pico endotérmico em $120{ }^{\circ} \mathrm{C}$ teve sua intensidade reduzida, indicando que parte da água que estava inicialmente presente na $\beta-C D$ foi deslocada pelo OEC, devido sua maior afinidade pela cavidade hidrofóbica da $\beta-\mathrm{CD},{ }^{14}$ sugerindo a formação do complexo. Aliado a este fenômeno, o pico exotérmico em $200{ }^{\circ} \mathrm{C}$ no termograma do

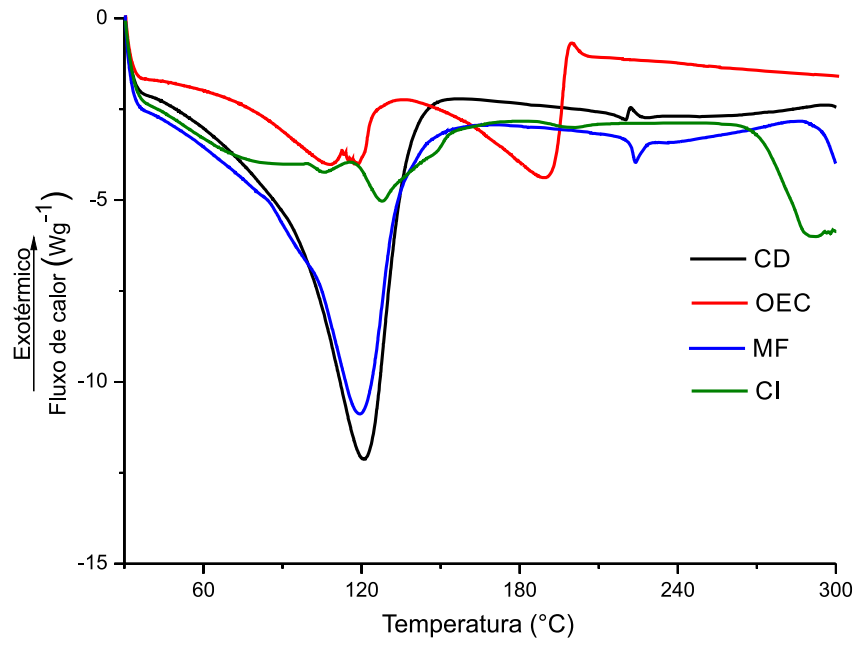

Figura 7. Termograma de DSC das Amostras de $\beta$-ciclodextrina ( $\beta$-CD), Mistura Física $(M F)$, Complexo de Inclusão (CI) e óleo essencial de Croton zehntneri $(O E C)$
OEC desaparece no CI, indicando uma maior estabilidade do OEC complexado quando comparado ao óleo livre.

É importante frisar que as ciclodextrinas vêm desempenhando um papel importante na Química Medicinal no que diz respeito à tecnologia de liberação controlada de fármacos, que representa atualmente uma das fronteiras da ciência envolvendo diferentes aspectos multidisciplinares do conhecimento. Os sistemas de liberação, frequentemente descritos como "drug delivery systems", oferecem inúmeras vantagens quando comparados a outros de dosagem convencional. ${ }^{42}$

\section{CONSIDERAÇÕES FINAIS}

O óleo essencial de Croton zehntneri foi analisado por CG-EM e seu complexo de inclusão com $\beta$-CD foi caracterizado por espectroscopia vibracional e por Calorimetria Exploratória Diferencial. Os resultados deste trabalho mostram claramente a formação do complexo de inclusão entre o óleo essencial de $C$. zenhtneri com $\beta-\mathrm{CD}$, pelo método de co-precipitação, a partir das análises de espectroscopia de IV e DSC, sendo possível inferir uma maior estabilidade do OEC complexado em comparação ao OEC livre, promovido pela sua introdução na cavidade fracamente polar da $\beta$-CD. Além disso, observou-se que existe compatibilidade da massa molecular desse fenilpropanóide com a da $\beta$-CD, permitindo assim a formação do complexo de inclusão, com um valor de constante de estabilidade compatível com uma possível aplicabilidade do material estudado, ampliando a perspectiva da avaliação da atividade farmacológica deste fenilpropanóide.

O principal componente identificado no óleo essencial em estudo foi o estragol. Esse componente tem sido aplicado na indústria alimentícia e cosmética, mas é suspeito de ser cancerígeno e genotóxico, como é indicado por um relatório da European Union, Committee on Herbal Medicinal Products. ${ }^{43} \mathrm{O}$ complexo de inclusão pode desempenhar um papel importante na Química Medicinal desse componente ou óleo essencial no que diz respeito à tecnologia de liberação controlada de um possível fármaco, em virtude das alterações nas propriedades físico-químicas, possibilitando a administração de uma dose reduzida e, consequentemente, a redução de efeitos colaterais.

\section{REFERÊNCIAS}

1. Oliveira, V. E.; Almeida, E. W. C.; Castro, H. V.; Edwards, H. G. M.; Dos Santos, H. F. L.; De Oliveira, F. C.; J. Phys. Chem. 2011, 115, 8511.

2. Craveiro, A. A.; Alencar, J. W.; Matos, F. J. A.; Andrade, C. H. S.; Machado, M. I. L.; Cien. Cult. 1978, 30, 326.

3. Morais, S. M.; Catunda-Junior, F. E. A. C.; Da Silva, A. R. A.; Neto, J. S. M.; Rondina, D.; Leal-Cardoso, J. H.; Quim. Nova 2006, 29, 907.

4. Simões, C. M. O.; Schenkel, E. P.; Gosmann, G.; Mello, J. C. P.; Mentz, L. A.; Petrovick, P. R.; Farmacognosia: da planta ao medicamento, $6^{\mathrm{a}}$ ed., Ed. da UFSC: Florianópolis, 2010.

5. Costa, J. G. M.; Rodrigues, F. F. G.; Angélico, E. C.; Pereira, C. K. B.; Souza, E. O. Caldas, G. F. R.; Silva, M. R., Santos, N. K. A.; Mota, M. L.; Santos, P. F.; Rev. Bras. Farmacogn. 2008, 18, 583.

6. Vincenzi, M. DE; Silanob, M.; Maialettia, F.; Scazzocchioa B.; Fitoterapia 2000, 71, 725 .

7. Shahat, A. A.; El-Barouty, G.; Hassan, R. A.; Hammouda, F. M.; Abdelrahman, F. H.; Saleh, M. A.; J. Environ. Sci. Health 2008, 43, 519.

8. Leal-Cardoso, J. H.; Matos-Brito, B. G.; Lopes-Junior, J. E. G.; VianaCardoso, K. V.; Sampaio-Freitas, A. B.; Brasil, R. O.; Coelho-de-Souza, A. N.; Albuquerque, A. A. C.; Braz. J. Med. Biol. Res. 2004, 37, 1193.

9. Batatinha, M. J.; De Souza-Spinosa, H.; Bernardi, M. M.; J. Ethnopharmacol. 1995, 45, 53.

10. Lyra, M. A. M.; Alves, L. D. S.; Fontes, D. A. F.; Soares Sobrinho, J. L.; Rolim Neto P. J.; Revista de Ciências Farmacêuticas Básica e Aplicada 2010, 31, 117. 
11. Guedes, F. L.; Alves, G. M. C.; Santos, F. L. A.; Lima, L. F.; Rolim, L. A.; Rolim Neto, P. J.; Rev. Bras. Farmacogn. 2008, 89, 220.

12. Veiga, F.; Pecorelli, C.; Ribeiro, L.; As ciclodextrinas em tecnologia farmacêutica, Minerva Coimbra: Coimbra, 2006.

13. Reineccius, T.; Reineccius, G.; Peppard, T. L.; J. Agric. Food Chem. 2005, 53, 388 .

14. Wang, J.; Cao Y.; Sun, B.; Wang, C.; Food Chem. 2011, 127, 1680.

15. Hedges, A. R.; Chem. Rev. 1998, 98, 2035.

16. Jiang, S.; Li, J.-N.; Jiang, Z.-T.; Eur. Food Res. Technol. 2010, 230, 543.

17. Wang, Y.; Jiang, Z.-T.; Li, R.; Eur. Food Res. Technol. 2009, 228, 865.

18. Tian, Z.-N.; Jiang, Z.-T.; Li, R.; Eur. Food Res. Technol. 2008, 227, 1001.

19. Fernandes, L. P.; Oliveira, W. P.; Sztatisz, J.; Szilágyi, I. M.; Novák, C. S.; J. Therm. Anal. Calorim. 2009, 95, 855.

20. Waleczek, K. J.; Cabral Marques, H. M.; Hempel, B.; Schmidt, P. C.; Eur. J. Pharm. Biopharm. 2003, 55, 247.

21. Zhan, H.; Jiang, Z.-T.; Wang, Y.; Li, R.; Dong, T.-S. Eur. Food Res. Technol. 2008, 227, 1507.

22. Cevallos, P. A.; Buera, M. P.; Elizalde, B. E.; J. Food Eng. 2010, 99, 70.

23. De Lima, S. G.; Moita Neto, J. M.; Costa, J. G. M. D.; Citó, A. M. G. L.; Reis, F. A. M.; J. Chil. Chem. Soc. 2009, 53, 1718.

24. Adams, R.P.; Identification of essential oil Components by gas chromatography/mass spectrometry, $4^{\text {th }}$ ed., Allured Publishing Corporation: Carol Stream, 2007.

25. Joulain, D.; Konig, W. A.; The atlas of spectral data of sesquiterpene hydrocarbons. E.B.-Verlag: Hamburg, 1998.

26. Sousa, E. O.; Silva, N. F.; Rodrigues, F. F. G.; Campos, A. R.; De Lima, S. G.; Costa, J. G. M.; Pharmacogn. Mag. 2010, 22, 78.

27. Bhandari, B. R.; D’arcy, B. R.; Bich, L. L. T.; J. Agric. Food Chem. 1998, 46, 1494.
28. Higuchi, T.; Connors, K. A.; Adv. Anal. Chem. Instrum. 1965, 4, 207.

29. Fernandes, L. P.; Oliveira, W. P.; Sztatisz, J.; Szilágyi, I. M.; Novák, C. S.; J. Therm. Anal. Calorim. 2002, 95, 855.

30. Loftsson, T.; Hreinsdóttir, D.; Másson, M.; Int. J. Pharm. 2005, 302, 18.

31. Rocha, M. S.; Dissertação de Mestrado, Universidade Federal do Piauí, Brasil, 2012.

32. Ribeiro, A. M.; Dissertação de Mestrado, Universidade do Porto, Portugal, 2008.

33. Oliveira, V. E.; Almeida, E. W. C.; Castro, H. V.; Edwards, H. G. M.; Dos Santos, H. F. L.;De Oliveira, F. C.; J. Phys. Chem. 2011, 115, 8511.

34. Szejtli, J.; Chem. Rev. 1998, 98, 1743.

35. Corti, G; Capasso, G; Maestrelli, F; Cirri, M; Mura, P.; J. Pharm. Biomed. Anal. 2007, 45, 480.

36. Macedo, L. F. O.; Dissertação de Mestrado, Universidade Federal de Sergipe, Brasil, 2010.

37. Larkin, P. J.; IR and Raman spectroscopy: principles and spectral interpretation, Elsevier: Oxford, 2011.

38. Thygesen, L. G.; Lokkey, M. M.; Micklander, E.; Engelsen, S. B.; Trends Food Sci. Technol. 2003, 14, 50.

39. Fini, A.; Ospitali, F.; Zoppetti, G.; Puccini, N.; Pharm. Res. 2008, 25, 2030.

40. Cunha-Filho, M. S. S.; Sá-Barreto, L. C. L.; Revista de Ciências Farmacêuticas Básica e Aplicada 2007, 28, 1.

41. Karathanos, V. T.; Mourtzinos, I.; Yannkopoulou, K.; Andrikopoulos, N. K.; Food Chem. 2007, 101, 652.

42. http://www.portaldosfarmacos.ccs.ufrj.br/resenhas_ciclodextrinas.html, acessada em Março 2013.

43. http://www.ema.europa.eu/pdfs/human/hmpc/13721205en.pdf, acessada em Junho 2013 